\title{
Selbstregulierung und ihre Krux mit der Publikation
}

Natassia Gili *

Werden Selbstregulierungen in das staatliche Recht einbezogen, meist durch einen Verweis, ist nicht nur die Zulässigkeit solcher Selbstregulierungen an sich fraglich. Es stellt sich auch die Frage, ob und falls ja, wie diese Selbstregulierungen zu publizieren sind. Sind sie dem vom Gesetz- bzw. Verordnungsgeber geschaffenen hoheitlichen Recht gleichgestellt, müsste sich deren Publikation nach dem Publikationsgesetz des Bundes richten. Behalten sie jedoch ihren privaten Charakter, sollte auf eine gesetzeskonforme Veröffentlichung verzichtet werden können. Problematisch ist dabei insbesondere die schwierige Einordnung einer Selbstregulierung unter das staatliche bzw. das private Recht.

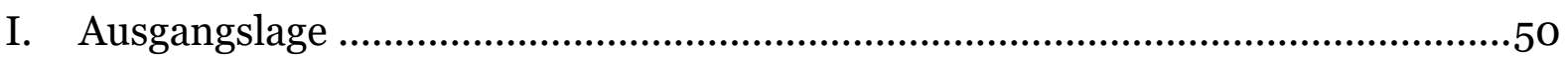

II. Staatlich gesteuerte Selbstregulierung ….....................................................50

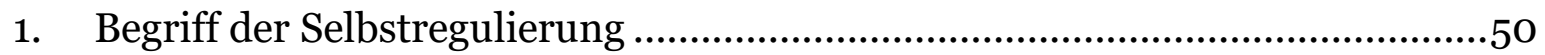

2. Rechtlicher Rahmen ........................................................................ 51

III. Übernahme von Selbstregulierungen in das staatliche Recht ...........................54

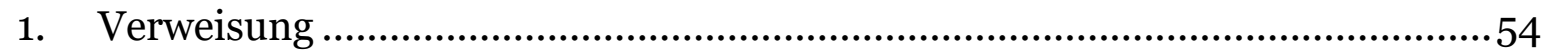

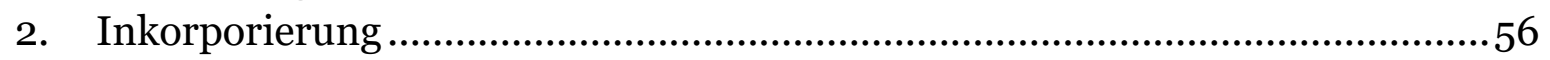

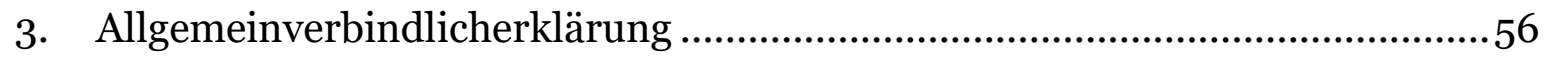

4. Branchenvereinbarung....................................................................... 56

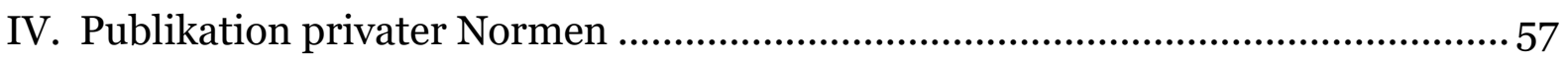

1. Publikation von Rechtsnormen im Allgemeinen .........................................57

2. Publikation durch Verweise im Sinne von Art. 5 PublG................................57

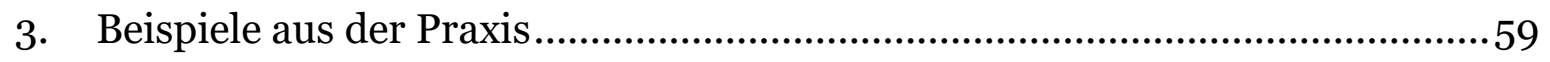

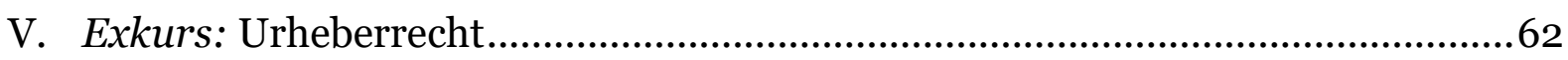

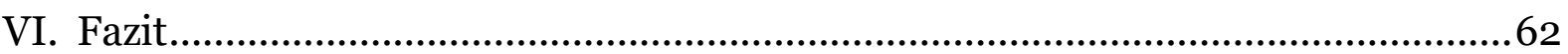

Zitiervorschlag: Natassia Gili, Selbstregulierung und ihre Krux mit der Publikation, in: sui-generis 2019, S. 49

URL: $\quad$ sui-generis.ch/92

DOI: $\quad$ https://doi.org/10.21257/sg.92

* Natassia Gili, Dr. iur., ist Gerichtsschreiberin am Bundesverwaltungsgericht (natassia.gili@bvger.admin.ch). Die Autorin dankt RA Dr. iur. Eliane Kohlbrenner und Dr. iur. Sibylle Dischler für die kritische Durchsicht und die wertvollen Hinweise. 


\section{Ausgangslage}

1 Je nach Rechtsanwendungsgebiet lässt sich in der Praxis ein Blick in die Fussnoten eines Gesetzes oder einer Verordnung kaum vermeiden. Nicht selten trifft man dabei Hinweise auf andere Normen und Regelwerke internationaler, nationaler, staatlicher oder privater Natur an. Insbesondere bei Normen, welche nicht aus der Feder des schweizerischen Gesetzgebers stammen, sondern von Privaten verfasst wurden, findet sich sodann die Information, wo die betreffende Norm konsultiert, abgerufen, bezogen oder gekauft werden kann. Ein kostenpflichtiger Download auf der Website des jeweiligen Normenerzeugers oder ein Gang zum zuständigen Bundesamt müssen von den Rechtsunterworfenen mithin in Kauf genommen werden. Wie verhält sich diese Praxis mit der dem demokratischen Rechtsstaat inhärenten Pflicht zur Publikation rechtsetzender Erlasse? Ist es doch unabdingbare Voraussetzung für die Anwendbarkeit und Verbindlichkeit einer Norm, dass diese für die Rechtsunterworfenen zugänglich ist und dass im Sinne der Rechtssicherheit und der Transparenz eine gewisse Vorhersehbarkeit und Erkennbarkeit der Rechtslage gewährleistet wird. ${ }^{1}$ So mutet es auf den ersten Blick etwas seltsam an, wenn rechtsanwendende Personen die sie verpflichtenden und berechtigenden Normen zunächst erwerben müssen, bevor sie diese einsehen und anwenden können. Um diese mittlerweile weit verbreitete Praxis rechtlich einordnen zu können,

Pierre Tschannen, Staatsrecht der Schweizerischen Eidgenossenschaft, 4. Aufl., Bern 2016, $\S 28 \mathrm{~N}$ 5; Benjamin Schindler, in: Bernhard Ehrenzeller/Benjamin Schindler/Rainer J. Schweizer/Klaus A. Vallender (Hrsg.), Die schweizerische Bundesverfassung, St. Galler Kommentar, Art. 1-93, 3. Aufl., Zürich 2014, Art. 5 N 38 f. muss in verschiedener Hinsicht differenziert werden. Die Frage nach der angestrebten Verbindlichkeit, dem Adressatenkreis und dem Preis-LeistungsVerhältnis stehen dabei im Fokus und sollen im Folgenden einer näheren Betrachtung unterzogen werden.

\section{Staatlich gesteuerte Selbstregulierung}

\section{Begriff der Selbstregulierung}

2 Werden private Normen in die staatliche Rechtsordnung miteinbezogen, ist von (staatlich gesteuerter) Selbstregulierung die Rede. Eine einheitliche Definition des Begriffs der Selbstregulierung fehlt in der schweizerischen Rechtsordnung. Entsprechend ist eine Abgrenzung und Differenzierung von anderen privaten Normierungsformen, wie bspw. Statuten eines Vereins, oder staatlichem Ausführungsrecht, z.B. Vollziehungsverordnungen, äusserst schwierig und teils auch kaum sinnvoll. ${ }^{2}$ Selbstregulierung umfasst jedoch m.E. im Mindesten eine Regelung einer privaten Interessengruppe, wobei diese Regelung primär privaten bzw. gesellschaftlichen Zwecken dient und dabei die - wie auch immer ausgestaltete staatliche Einflussnahme beschränkt oder gar ausgeschlossen ist. Ausgehend von der Privatautonomie ist die sogenannte reine Selbstregulierung einer privaten Selbstverwaltung gleichzusetzen, die lediglich Wirkung inter partes zu erzeugen vermag und gänzlich ohne hoheitlichen Einfluss auskommt. Sobald der Staat auf die Selbstregulierung ein-

\footnotetext{
2 Vgl. anschaulich zu den Reglementen der SIX Swiss Exchange Philippe Borens, in: Giovanni Biaggini/Isabelle Häner/Urs Saxer/Markus Schott (Hrsg.), Fachhandbuch Verwaltungsrecht, Zürich/Basel/Genf 2015, N 4.40.
} 
wirkt, wobei diese Einflussnahme bis hin zur paritätischen Kooperation von Staat und Privaten reichen kann, ist von staatlich gesteuerter Selbstregulierung die Rede. ${ }^{3}$ Staatlich gesteuerte Selbstregulierung kann in der Praxis die unterschiedlichsten Ausprägungen annehmen. Dabei entspricht sie stets dem demokratischliberalen Verständnis des heutigen Gewährleistungsstaates 4 . Zugleich ist sie Folge des sich weiterhin haltenden Vorwurfs der staatlichen Überregulierung und mithin eine Form der Deregulierung.5

\section{Rechtlicher Rahmen}

3 Mangels einer expliziten Rechtsgrundlage, insbesondere im Hinblick auf die $\mathrm{Zu}-$ lässigkeit von Selbstregulierungen, werden zumeist die Voraussetzungen von Art. 164 BV und Art. 178 Abs. 3 BV sinngemäss herangezogen.

\section{a) Art. $164 \mathrm{BV}$}

4 Im Sinne des allgemeinen Legalitätsprinzips nach Art. 5 Abs. 1 BV setzt der in Art. 164 Abs. 1 BV statuierte Gesetzesvorbehalt für den Erlass wichtiger Bestimmungen die Form des Bundesgesetzes voraus. ${ }^{6}$ Für wichtige Inhalte ${ }^{7}$ werden ei-

Siehe zum Ganzen Natassia Gili, Staatlich gesteuerte Selbstregulierung am Beispiel der medizinischethischen Richtlinien der Schweizerischen Akademie der Medizinischen Wissenschaften (SAMW), Zürich/Basel/Genf 2017, S. $6 \mathrm{ff}$.

4 Zum Begriff des Gewährleistungsstaates siehe Gili (Fn. 3), S. 12 f. m.w.H.

5 Gili (Fn. 3), S. 35 ff.

6 Tschannen (Fn. 1), § 45 N 8, der den Begriff «gesetzespflichtiger Inhalt» vorzieht; auch «materieller Gesetzesvorbehalt» genannt, siehe René Rhinow/Markus Schefer/Peter Uebersax, Schweizerisches Verfassungsrecht, 3. Aufl., Basel 2016, N 2720 ff.; Giovanni Biaggini, BV Kommentar, 2. Aufl., Zürich 2017, Art. 164 N 3. ne hohe Normstufe (Gesetz im formellen Sinne) sowie eine hohe Normdichte (Erfordernis des Rechtssatzes) gefordert. ${ }^{8}$ Daraus ergibt sich im Gegenzug ein Delegationsverbot für wichtige Inhalte (Art. 164 Abs. 2 i.V.m. Abs. 1 BV). Die Voraussetzungen der Gesetzesdelegation (eigentlich: Delegation von Rechtsetzungsbefugnissen) sind sodann in Art. 164 Abs. 2 BV verankert.9 Vom Grundsatz, dass die Gesetzgebungskompetenz bei der Bundesversammlung liegt, kann abgewichen werden, sofern die Delegation nicht durch die Bundesverfas-

7 Das Kriterium der Wichtigkeit ist hinsichtlich seines Inhalts, seiner Tragweite und seiner Abgrenzung zum Begriff «grundlegend» in Art. 164 Abs. 1 Satz 1 BV umstritten und weitgehend der politischen Wertung unterworfen, siehe Gili (Fn. 3), S. 43 f. m.w.H. Immerhin führt Art. 164 Abs. 1 BV Beispiele grundlegender Normkategorien auf, siehe kritisch dazu Biaggini (Fn. 6), Art. 164 N 5. Zusätzliche Abhilfe schaffen zudem die durch Lehre und Rechtsprechung entwickelten Hilfskriterien. Die Notwendigkeit einer Regelung in einem formellen Gesetz beurteilt sich demnach nach der Zahl der geregelten Verhaltensalternativen, der Grösse des Adressatenkreises, wie stark in die (Grund-)Rechtsstellung der Betroffenen eingegriffen wird (Intensität einer Regelung), den finanziellen Auswirkungen für Staat und Private, der Umstrittenheit bzw. Akzeptanz durch die Rechtsunterworfenen, der Bedeutung für die politische Willensbildung, die Behördenorganisation oder das Verfahren; siehe Biaggini (Fn. 6), Art. 164 N 6; Georg Müller/Felix Uhlmann, Elemente einer Rechtssetzungslehre, 3. Aufl., Zürich/Basel/Genf 2013, N 230; Gesetzgebungsleitfaden, Leitfaden für die Ausarbeitung von Erlassen des Bundes, Bundesamt für Justiz, 3. Aufl., Bern 2007, N 595.

8 Pierre Tschannen, in: Bernhard Ehrenzeller/Benjamin Schindler/Rainer J. Schweizer/Klaus A. Vallender (Hrsg.), Die schweizerische Bundesverfassung, St. Galler Kommentar, Art. 94-197, 3. Aufl., Zürich 2014, Art. 164 N 6.

9 In Art. 164 Abs. 2 BV nicht ausdrücklich erwähnt sind die durch das Bundesgericht für die kantonale Ebene entwickelten „Delegationsgrundsätze“ (siehe oben Fn. 7), wobei anzunehmen ist, dass diese in Art. 164 Abs. 2 BV stillschweigend mitgemeint sind, siehe Biaggini (Fn. 6), Art. 164 N 13; ähnlich Tschannen (Fn. 8), Art. 164 N 34; siehe auch BGE 128 I 113 E. 3.c) S. 121; BGE 130 I 26 E. 5 S. $43 \mathrm{f}$. 
sung ausgeschlossen ist ${ }^{10}$, sich der Regelungsbereich auf eine bestimmte Materie beschränkt ${ }^{11}$, und die Delegationsnorm selbst sowie die Grundzüge der delegierten Materie in einem formellen Gesetz ${ }^{12}$ enthalten sind. Über den Adressatenkreis solcher Delegationsbefugnisse schweigt sich die Verfassung bedauerlicherweise aus. Ob Art. 164 Abs. 2 BV - welcher in der Praxis insbesondere Grundlage für die Delegation von Rechtsetzungsbefugnissen an die Kantone sowie an den Bundesrat und dessen Departemente bildet auch für die Delegation von Rechtsetzungsbefugnissen an Private herangezogen werden kann, ist in der Lehre weitgehend umstritten. M.E. ist die Delegation von Rechtsetzungsbefugnissen an Private zulässig, sofern sie im Sinne von Art. 164 Abs. 2 BV durch die Verfassung nicht ausgeschlossen ist und die übrigen durch Verfassung und Rechtsprechung geschaffenen Voraussetzungen erfüllt sind.13 Das Erfordernis eines expliziten Verfassungsvorbehalts $^{14} \mathrm{zu}$ bejahen, würde nach der hier vertretenen Meinung zu weit gehen.

10 Biaggini (Fn. 6), Art. 164 N 12.

11 Verbot der Pauschaldelegation.

12 D.h. in einem referendumspflichtigen, rechtsetzenden Erlass, vgl. Art. 163 i.V.m. Art. 141 BV.

13 Ebenso Biaggini (Fn. 6), Art. 164 N 11; Ulrich Häfelin/Walter Haller/Helen Keller/Daniela Thurnherr, Schweizerisches Bundesstaatsrecht, 9. Aufl., Zürich/Basel/Genf 2016, N 1890; Rhinow/Schefer/ Uebersax (Fn. 6), N 2739.

14 Gefordert u.a. von Jean-François Aubert/Pascal Mahon, Petit commentaire de la Constitution fédérale de la Confédération suisse du 18 avril 1999, Zürich 2003, Art. 164 N 40; Christoph Errass, Kooperative Rechtssetzung, Zürich/St. Gallen 2010, N 313; siehe auch Pascal Zysset, Selbstregulierung im Finanzmarktrecht, Zürich/Basel/ Genf 2017, N 593, unter Bezugnahme auf die historische Auslegung von Art. 164 BV.

\section{b) Art. 178 Abs. 3 BV}

5 In Art. 178 Abs. 3 BV wird im Sinne der Dezentralisierung die Auslagerung von Verwaltungsaufgaben auf andere, ausserhalb der Bundesverwaltung stehende staatliche Aufgabenträger geregelt. Zur Auslagerung von Verwaltungsaufgaben ist eine Ermächtigung durch das Gesetz erforderlich, wobei die Anforderungen an die formell-gesetzliche Grundlage nicht in allen Anwendungsbereichen gleich hoch sind und sich nicht alle Verwaltungsaufgaben für eine Dezentralisierung eignen. ${ }^{15}$ Des Weiteren muss die Auslagerung im öffentlichen Interesse liegen (Art. 5 BV) und der Rechtsschutz (Art. 29a BV) sowie die Funktionstauglichkeit des neuen Rechtsträgers müssen längerfristig sichergestellt sein. Schliesslich ist eine staatliche Aufsicht erforderlich und die Aufgabenträger haben sich an die Grundrechte zu halten (Art. 35 Abs. 2 BV). Im Hinblick auf die Frage der Selbstregulierung sind jedoch nicht die Voraussetzungen an sich problematisch, sondern die vorgelagerte Frage, was unter einer Verwaltungsaufgabe $\mathrm{zu}$ verstehen ist; dies wird in der Verfassung jedoch nicht näher definiert. Je enger der Begriff der Verwaltungsaufgabe ausgelegt wird, desto weniger ist die Subsumierung der Übertragung von Rechtsetzungsbefugnissen an Private unter Art. 178 Abs. 3 BV möglich. ${ }^{16}$ M.E. gehen Rechtsetzungsbefugnisse in ihren Auswirkungen weiter als die blosse Übertragung einer Verwaltungsaufgabe bzw. die Vollzugsdelegation. Als Grundlage für die staatlich

15 Giovanni Biaggini, in: Bernhard Ehrenzeller/Benjamin Schindler/Rainer J. Schweizer/Klaus A. Vallender (Hrsg.), Die schweizerische Bundesverfassung, St. Galler Kommentar, Art. 94-197, 3. Aufl., Zürich 2014, Art. 178 N 28 m.w.H.

16 Siehe dazu Gili (Fn. 3), S. 52 f. 
gesteuerte Selbstregulierung kann, je nach deren Ausgestaltung, sowohl Art. 164 BV als auch Art. 178 Abs. 3 BV dienen.

\section{c) Rechtsprechung des Bundesgerichts}

6 Das Bundesgericht hat sich bisher erst in wenigen Fällen mit den Voraussetzungen der Zulässigkeit von Selbstregulierungen befasst:

7 In BGE 143 II 162 hielt es beschränkt auf den Anwendungsbereich des $\mathrm{GwG}^{17}$ fest, dass im Rahmen der Selbstregulierung der Erlass technischer Vorschriften zulässig ist, nicht jedoch die Einführung neuer Pflichten. ${ }^{18}$

In BGE 143 II 518 zum Inverkehrbringen von Produkten nach dem THG ${ }^{19}$ und dem PrSG ${ }^{20}$ stellte es fest, dass die beiden Gesetze auf dem Grundsatz der gesteuerten Selbstregulierung basieren würden, wonach der Staat lediglich den Rahmen setze. Innerhalb dieses Rahmens obliege die Regulierung den Privaten. Auch im Rahmen des Vollzugs sei es nicht Aufgabe des Staates, Lösungen vorzuschlagen, da ansonsten das System der gesteuerten Selbstregulierung qua Vollzug unterlaufen werden würde. ${ }^{21}$
9 In BGE 136 I 316 hat das Bundesgericht bei der Beurteilung einer SIA22-Norm Klärungen in Bezug auf die Verweisungspraxis vorzunehmen versucht und dabei festgehalten, dass statische Verweise, d.h. Verweise auf eine bestimmte Fassung einer Norm, ${ }^{23}$ grundsätzlich als unproblematisch anzusehen seien. Dynamische Verweise hingegen, mit welchen faktisch Rechtsetzungsbefugnisse an Private delegiert würden, seien nur zulässig, wenn eine Verfassungsnorm die Privaten als Rechtsetzungssubjekte vorsehe und den notwendigen gesetzlichen Übertragungsakt bezeichne. ${ }^{24}$ Wird die Setzung von Normen delegiert, die Gegenstand einer Vollziehungsverordnung bilden, handle es sich dagegen funktional um Verwaltung. Dabei würden nicht Rechtsetzungsbefugnisse delegiert, sondern lediglich Verwaltungsaufgaben im Sinne von Art. 178 Abs. 3 BV auf Private übertragen. 25

10 Diese Entscheide zeigen, dass Selbstregulierungen durchaus Einzug in den Rechtsalltag gehalten haben und es in der Praxis vermehrt zu Auslegungsund Abgrenzungsschwierigkeiten kommen kann. Wünschenswert wäre, wenn sich das Bundesgericht nicht nur beschränkt auf die im Einzelfall untersuchte Norm, sondern sich auch in allgemeiner Weise zur Zulässigkeit der staatlich gesteuerten Selbstregulierung äussern würde.

22 Schweizerischer Ingenieur- und Architektenverband.

23 S. zum Instrument der Verweisung sogleich III. 1.

24 S. auch BGE 133 V 96 E. 4.4.5 S. 102; BGE 123 I 112 E. 7 S. $124 \mathrm{ff}$.

25 BGE 136 I 316 E. 2.4.1 S. 319 f.; Felix Uhlmann, Entwicklungen im Verwaltungsrecht, SJZ 107/2011 S. 414 ff., 414 f.; ders., «Die Normen können bei ... bezogen werden» - Gedanken zur Publikation und Verbindlichkeit privater Normen, LeGes 2013/1, S. 89 ff., 94 (zit. Uhlmann, Publikation). 


\section{d) Zwischenfazit}

11 Grundsätzlich ist die staatlich gesteuerte Selbstregulierung als zulässig zu erachten. Mangels einer expliziten Rechtsgrundlage wird im Einzelfall zunächst zu prüfen sein, ob eine bestimmte Norm einen öffentlich-rechtlichen oder privaten Charakter aufweist. Wird sie dem öffentlichen Recht zugeordnet, sind zwingend die oben genannten, im Rahmen von Art. 164 BV geltenden Voraussetzungen einzuhalten. Und auch umgekehrt wird eine Norm nur dem öffentlichen Recht zugeordnet werden können, wenn sie die durch Verfassung und Rechtsprechung entwickelten Voraussetzungen einhält. Wird also eine ursprünglich private Regulierung auf die materielle Ebene des staatlichen Rechts gehoben und als öffentlich-rechtliche Norm qualifiziert, kommt ihr dieselbe Verbindlichkeit zu wie dem durch den hoheitlichen Gesetzgeber geschaffenen Recht. Folglich müsste sie auch auf dieselbe Weise publiziert und zugänglich gemacht werden.

\section{III. Übernahme von Selbstregulierun- gen in das staatliche Recht}

\section{Verweisung}

\section{a) Verweisung als rechtsetzungs- technisches Instrument}

Eine mittlerweile etablierte Praxis um private Normen in das staatliche Recht zu überführen und ihnen Verbindlichkeit zu verleihen, stellt die Verweisung dar. Die Verweisung an sich ist ein rechtsetzungstechnisches Instrument. Der Gesetzgeber verweist dabei in seinem Gesetz oder seiner Verordnung (Verweisungssubjekt) auf eine andere, bereits bestehende Norm (Verweisungsobjekt).
Dabei kann der Gesetzgeber nicht nur auf seine eigenen staatlichen Normen verweisen, sondern auch auf private Normen, die durch die Verweisung zu einem integralen, materiellen Bestandteil des staatlichen Erlasses werden. Auf diese Weise können faktisch Rechtsetzungsbefugnisse auf Private übertragen werden, weswegen die Voraussetzungen der Gesetzesdelegation nach Art. 164 Abs. 2 BV $\mathrm{zu}$ beachten sind. ${ }^{26}$ Problematisch ist dabei sicherlich das Erfordernis einer gesetzlichen Grundlage, zumal in der Praxis die meisten Verweise auf private Regelwerke erst auf Verordnungsstufe zu finden sind. Je nachdem, welchen Charakter das Verweisungsobjekt aufweist und welche Verbindlichkeit es anstrebt, genügt eine Grundlage in einer Verordnung jedoch nicht. ${ }^{27}$

13 Unterschieden wird zwischen normativen und informativen, direkten und indirekten, dynamischen und statischen sowie zwischen Binnen- und Aussenverweisen. ${ }^{28}$ Insbesondere die Unterscheidung von dynamischen und statischen Verweisungen ist aus rechtsstaatlicher Sicht interessant. So wird von einer statischen Verweisung gesprochen, wenn auf eine bestimmte Fassung einer Norm verwiesen wird, z.B. mittels des Zusatzes «in der Fassung vom 01.01.2019». Auf diese Weise wird eine Abänderung des Verweisungsobjekts ohne Zutun des Gesetzgebers verhindert. Entsprechend findet durch den statischen Verweis keine eigentliche Rechtsetzungsdelegation im Sinne von Art. 164 Abs. 2 BV statt, weswegen auch die Anforderungen herabzu-

26 Siehe oben II. 2.1.

27 Uhlmann, Publikation (Fn. 25), S. 95.

28 Siehe zur Definition der Begriffspaare Müller/ Uhlmann (Fn. 7), N 360 ff. 
setzen sind. ${ }^{29}$ Dynamisch sind Verweisungen hingegen, wenn das Verweisungsobjekt, sprich die Selbstregulierung, in der jeweils aktuell geltenden Fassung verbindlich ist. Dies führt dazu, dass die private Norm ohne Zutun des Gesetzgebers verändert werden kann, was faktisch einer Blankoermächtigung für die Privaten gleichkommt. Dynamische direkte Verweisungen sind daher, wie auch bereits vom Bundesgericht festgestellt3o, heikel.

14 In der Praxis wird des Öfteren auf «anerkannte Regeln» (der Baukunde, der Technik, der Kunst usw.) oder auf den «Stand der Wissenschaft» verwiesen. Solche indirekten, rein informativen Verweise sind nicht als eigentliche Verweise im oben genannten Sinne zu qualifizieren, sondern weisen lediglich auf einen bestimmten Standard und gerade nicht auf ein spezifisches privates Normenwerk hin. Durch die blosse Erwähnung eines solchen Standards in einem Gesetz oder einer Verordnung, wobei Inhalt und Tragweite des Standards der Auslegung durch die Rechtsanwendung unterworfen sind, werden diese jedoch nicht zu verbindlichem Recht. ${ }^{1}$

$29 \overline{\text { Siehe auch Arnold Marti, Aufgabenteilung zwi- }}$ schen Staat und Privaten auf dem Gebiet der Rechtsetzung - Ende des staatlichen Rechtsetzungsmonopols?, AJP 2002, S. 1154 ff., 1161.

30 BGE 136 I 316 E. 2.4.1 S. $319 \mathrm{f}$.

31 Siehe Gili (Fn. 3), S. 101, 107 f.; Marius Roth, Die Veröffentlichung von Rechtsnormen in der Schweiz，Zürich/St. Gallen 2012，S. 182; siehe auch Uhlmann, Publikation (Fn. 25), S. 96 ff., der solche Hinweise unter die Verwaltungspraxis bzw. die blosse Konkretisierung staatlichen Rechts subsumiert; siehe Nadine Mayhall, Aufsicht und Staatshaftung, Zürich/Basel/Genf 2008, S. 178, die von «Technikklauseln» spricht.

\section{b) Rechtsnormqualität der Verweisung}

15 Durch die Verweisung wird das ursprünglich private Recht in die Sphäre des staatlichen Rechts gebracht. Ob die privaten Normen auf diese Weise zu staatlichem Recht werden oder nicht, ist umstritten. Dem Verweisungsobjekt die Qualifizierung als staatliches Recht ganz abzuerkennen, wäre wohl am einfachsten und würde zudem die Problematik der Publikation geschickt umgehen: Die privaten Normen, auf die verwiesen wird, würden privat bleiben und daher keiner Publikation bedürfen. ${ }^{2}$ Diese Ansicht greift m.E. zu kurz. Die Absicht des Gesetzgebers bei der Verwendung von Verweisungen ist nicht, den Privaten im Sinne der Privatautonomie freien Regelungsspielraum zu lassen, zumal ihm hierfür andere Instrumente wie die Privatisierung zur Verfügung stehen. Er verzichtet zwar zugunsten privater Regulierungen auf den Erlass einer eigenen staatlichen Regelung, nimmt aber durch den Verweis wieder Bezug auf die private Norm und führt diese zurück in seinen Einflussbereich. Dies entspricht gerade dem Charakter der staatlich gesteuerten Selbstregulierung, wonach Private eine Selbstregulierung erlassen können, dabei jedoch weiterhin dem, wie auch immer ausgestalteten, staatlichen Einfluss unterworfen sind. Insofern ist eine Selbstregulierung, auf die das staatliche Recht verweist, nicht privater Natur.

Davon auszugehen, dass private Normen qua Verweis zu staatlichem Recht werden,33 würde hingegen auch zu weit gehen. Staatlich gesteuerte Selbstregulierungen befinden sich gerade im Schnittbereich

32 Marti (Fn. 29), S. 1161.

33 So wohl Uhlmann, Publikation (Fn. 25), S. 94. 
von staatlichem und privatem Recht und können nicht gänzlich dem einen oder dem anderen Rechtskreis zugeordnet werden.34 Nach der hier vertretenen Meinung werden somit die privat entstandenen Normen durch die Verweisung vom Staat anerkannt und erhalten eine gewisse Verbindlichkeit. Dennoch untersteht die staatlich gesteuerte Selbstregulierung nicht per se denselben rechtsstaatlichen Prinzipien wie das vom Gesetzgeber erlassene Recht. Wie so oft, wenn eine klare Rechtsgrundlage fehlt, wird im Einzelfall zu beurteilen sein, ob ein Verweisungsobjekt mehrheitlich staatliches oder privates Recht darstellt.

\section{Inkorporierung}

17 Sofern private Normen in ihrer Gesamtheit, mehr oder weniger wörtlich, in einen staatlichen Erlass übernommen werden, ist von Inkorporation oder Inkorporierung die Rede. Die ursprünglich private Natur der Normen ist in der Folge nicht mehr als solche erkennbar und die Normen sind dem hoheitlichen Recht sowohl materiell als auch formell gleichgestellt. Insofern stellen sich auch keine Fragen in Bezug auf deren Verbindlichkeit oder die Publikation. 35

\section{Allgemeinverbindlicherklärung}

18 Durch die Allgemeinverbindlicherklärung werden primär in den Bereichen des Mietrechts (Art. 109 Abs. 2 BV) und des Arbeitsrechts (Art. 110 Abs. 1 lit.d BV) private Normen als allgemeinverbindlich, d.h. für weitere Akteure desselben Wirtschaftszweigs als verbindlich, erklärt.

34 Ulrich Häfelin/Georg Müller/Felix Uhlmann, Allgemeines Verwaltungsrecht, 7. Aufl., Zürich/ St. Gallen 2016, N 251; Mayhall (Fn. 31), S. 180, 185 f. m.w.H.

35 Siehe auch die Beispiele in Gili (Fn. 3), S. 96 f.
Aufgrund des relativ starken Eingriffs in die Privatautonomie der Betroffenen gelten für die Allgemeinverbindlicherklärungen zusätzliche verfassungsrechtliche Voraussetzungen. Die Problematiken um Verbindlichkeit und Publikation sind begrenzt, da die Wirkung von Allgemeinverbindlicherklärungen von Gesamtarbeitsverträgen in Art. 5 AVEG ${ }^{36}$ und deren Publikation in Art. 14 AVEG explizit geregelt sind; für den Bereich des Mietrechts sind die entsprechenden Vorgaben in Art. 5 und 11 des Bundesgesetzes über Rahmenmietverträge und deren Allgemeinverbindlicherklärung $37 \mathrm{zu}$ finden. ${ }^{8} 8$

\section{Branchenvereinbarung}

19 Formulierungen, die den Adressaten z.B. dazu auffordern, «bestimmte Vorschriften soweit möglich und notwendig beim Vollzug zu beachten», weisen auf Branchenvereinbarungen hin. Diese sind grundsätzlich nur für die betreffenden Mitglieder rechtsverbindlich, erhalten aber durch die Anerkennung durch den Bundesrat oder einer anderen Verwaltungsbehörde eine gewisse, wenn auch beschränkte Verbindlichkeit, indem sie zu Mindeststandards erhoben werden und dem Gesetzgeber als Auslegungshilfe dienen. Da Branchenvereinbarungen trotz Anerkennung durch eine staatliche Instanz dem privaten Recht zugehörig bleiben und keine generellabstrakten Rechtswirkungen zu erzeugen vermögen, ergeben sich auch hier kaum Schwierigkeiten im Hinblick auf deren Publikation und Verbindlichkeit. 39

36 Bundesgesetz vom 28. September 1956 über die Allgemeinverbindlicherklärung von Gesamtarbeitsverträgen (AVEG; SR 221.215.311).

37 Bundesgesetz vom 23. Juni 1995 über Rahmenmietverträge und deren Allgemeinverbindlicherklärung (SR 221.213.15).

38 Mayhall (Fn. 31), S. $181 \mathrm{ff}$.

39 Gili (Fn. 3), S. 106 f.; Luzian Odermatt, Gesetzge- 


\section{Publikation privater Normen}

\section{Publikation von Rechtsnormen im Allgemeinen}

20 Erst durch die Publikation und somit ermöglichte Kenntnisnahme durch die Rechtsunterworfenen entfalten die in den Rechtstexten statuierten Rechte und Pflichten ihre Wirkung (Art. 8 Abs. 1 PublG). Die Publikation von Erlassen des Bundes $4^{\circ}$ erfolgt gestützt auf das Publikationsgesetz ${ }^{41}$. Art. 2 PublG nennt dabei die Erlasse des Bundes, welche in der Amtlichen Sammlung (AS) zu veröffentlichen sind. Im zweiten Halbsatz von Art. 2 lit. e PublG wird ferner die Publikation in der AS vorgesehen für übrige rechtsetzende Erlasse «von Organisationen und Personen des öffentlichen oder des privaten Rechts ${ }^{42}$, die mit Verwaltungsaufgaben betraut sind, jedoch nicht der Bundesverwaltung angehören» (siehe Art. 178 Abs. 3 BV). Von Art. 2 lit. e PublG sind dem Wortlaut nach jedoch nur rechtsetzende Erlasse erfasst. Demnach fällt eine Veröffentlichung nur dann unter Art. 2 lit. e PublG, wenn mit der Übertragung von Verwaltungsaufgaben auch Rechtsetzungsbefugnisse im Sinne von Art. 164 Abs. 2 BV auf die Organisationen und Personen des öffentlichen und privaten Rechts delegiert werden. Zur Publikation privater Normen schweigt sich das Publikationsgesetz ansonsten aus.

bungsmethodische Strategien der Übernahme privater Normen, LeGes 2006/3, S. 85 ff., $87 \mathrm{ff}$.

40 Für die Kantone siehe Roth (Fn. 31), S. 26 ff., $31 \mathrm{ff}$.

41 Bundesgesetz vom 18. Juni 2004 über die Sammlungen des Bundesrechts und das Bundesblatt, (PublG; SR 170.512).

42 Art. 6 Abs. 3 erster Satz RVOV (Regierungs- und Verwaltungsorganisationsverordnung vom 25. November 1998 [RVOV; SR 172.010.1]); bspw. in der Form von privatrechtlichen und spezialgesetzlichen Aktiengesellschaften (SBB), öffentlich-rechtlichen oder privatrechtlichen Stiftungen (Pro Helvetia) oder öffentlich-rechtlichen Anstalten (SUVA).

\section{Publikation durch Verweise im Sinne von Art. 5 PublG}

21 Vom Grundsatz, dass jeder Erlass in seiner Gesamtheit in der AS bzw. auf der Onlineplattform des Bundes (sog. Publikationsplattform) zu publizieren ist, kann nach Massgabe von Art. 5 Abs. 1 PublG ausnahmsweise abgewichen werden, wenn der publikationspflichtige Rechtstext wegen seines «besonderen Charakters» zur Veröffentlichung als Ganzes ungeeignet ist.43 Dies ist namentlich der Fall, wenn sich der Text nur an einen kleinen Adressatenkreis richtet (lit. a), wenn er technischer Natur ist und sich nur an Fachleute wendet (lit. b), wenn er in einem Format veröffentlicht werden muss, das sich für die Veröffentlichung in der AS nicht eignet (lit. c) oder wenn ein Bundesgesetz oder eine Verordnung die Veröffentlichung ausserhalb der AS anordnet (lit. d). In die Kategorien von lit. a und b fallen insbesondere technische Normen, bspw. Sicherheitsbestimmungen im Bereich des Personentransports, im Baubereich oder im Medizinalbereich.44 Unter lit. c fallen bspw. Datenbanken45, die sich in der Form eines Rechtstextes oder eines Anhanges nicht publizieren lassen. Weisen also Rechtstexte im Sinne der Art. 2-4 PublG (Erlasse des Bundes, völkerrechtliche Verträge und Konkordate) einen solchen «besonderen Charakter» nach Art. 5 Abs. 1 PublG auf, wird lediglich

43 Roth (Fn. 31), S. 6o f.

44 So z.B. die Verordnung vom 12. Januar 1987 über die Sicherheitsanforderungen an Umlaufbahnen mit festen Klemmen (Sesselbahnverordnung; SR 743.121.2), oder die Verordnung vom 17. Juni 1991 über die Sicherheitsanforderungen an Standseilbahnen (Standseilbahnverordnung; SR 743.121.6); ebenso die Pharmacopoea Helvetica, siehe sogleich IV. 3.4.

45 Z.B. die in der NHV (Verordnung vom 16. Januar 1991 über den Natur- und Heimatschutz, [NHV; SR 451.1]) vorgesehenen «Inventare». 
ein Verweis, sprich deren Titel und Fundstelle bzw. Bezugsquelle, in die AS aufgenommen.

Art. 5 Abs. 2 PublG sieht eine Ausnahme von der Veröffentlichung in der AS für Rechtstexte vor, die von einem externen Publikationsorgan herausgegeben werden. So sind Rechtstexte, «die in einem anderen in der Schweiz unentgeltlich zugänglichen Publikationsorgan veröffentlicht sind», ebenfalls als Verweis, d.h. mit Titel sowie der Fundstelle in diesem Organ oder der Bezugsstelle, in die AS aufzunehmen. In erster Linie geht es hierbei um Rechtsakte der EU46 und private Normen, die durch das Schweizerische Recht für anwendbar erklärt wurden.47 Die Botschaft nennt ausdrücklich staatliche, supranationale und private Trägerschaften und weist darauf hin, dass es insbesondere bei Normen privater Organisationen zu Schwierigkeiten in Bezug auf die geforderte Unentgeltlichkeit und den urheberrechtlichen Schutz ${ }^{48}$ kommen kann.

Art. 14 Abs. 3 der Publikationsverordnung49 führt hierzu konkretisierend aus, dass bei einer Publikation durch Verweis im Sinne von Art. 5 Abs. 2 PublG Folgendes in der AS aufgeführt sein muss: die Inter-

$46 \overline{\text { Siehe zur Problematik der Verweisungen auf EU- }}$ Recht Clemens Locher, Redaktionelle und gesetzestechnische Probleme der Verweisung auf EURecht im Landesrecht, LeGes 2010/1, S. 87 ff.; Bundeskanzlei, Faustregeln für die Umsetzung von EU-Recht in schweizerisches Recht, 20.09.2017, m.w.H.

47 Botschaft zur Änderung des Publikationsgesetzes (Primatwechsel von der gedruckten zur elektronischen Version der amtlichen Veröffentlichungen) vom 28. August 2013 (BBl 20137057 ff. [zit. Botschaft PublG]), S. 7078 .

48 Siehe sogleich V.

49 Verordnung vom 7. Oktober 2015 über die Sammlungen des Bundesrechts und das Bundesblatt (PublV; SR 170.512.1). netadresse, über die der Text eingesehen oder bezogen werden kann (lit. a), die federführende Behörde oder allenfalls andere Stellen, bei welchen der Text unentgeltlich eingesehen werden kann (lit.b), sowie die Postadresse, bei welcher der Text bezogen werden kann, wenn die betreffende Stelle über keine Internetadresse verfügt (lit. c). Im Umkehrschluss ist folglich anzunehmen, dass bei Publikationen durch Verweise im Sinne von Art. 5 Abs. 2 PublG die Zugänglichkeit über eine Internetseite sowie bei der Behörde bzw. beim privaten Anbieter gewährleistet sein muss, jedoch lediglich bei Letzterem der $\mathrm{Zu}$ gang kostenlos zu sein hat.50 So führt auch die Botschaft zum Publikationsgesetz aus, dass die Verwendung eines Verweises nach Art. 5 Abs. 2 PublG nur zulässig ist, wenn die Kostenlosigkeit der Konsultation der Texte gewährleistet ist. Auch aus dem in der Botschaft angeführten Beispiel der Normen, die bei der Schweizerischen Normenvereinigung (SNV) $\mathrm{zu}$ beziehen sind, ${ }^{1}$ ist $\mathrm{zu}$ entnehmen, dass die gesetzlichen Anforderungen erfüllt sind, solange die Normen bei der Geschäftsstelle einsehbar sind. Ein kostenloser Online-Zugang wird nicht verlangt. ${ }^{2}$ Anders als noch in der Publikationsverordnung vom 17. November $2004^{53}$ wird ausserdem keine Veröffentlichung in den erforderlichen Amtssprachen vorausgesetzt, so dass sich die Problematik der nur in englischer Sprache verfügbaren internationalen bzw. EU-rechtlichen Normen oder der nur in einer Landes-

$50 \overline{\text { In der Fassung vom 17. November } 2004 \text { hatte die }}$ sachlich zuständige Behörde noch dafür zu sorgen, dass eine «allfällige Online-Version unentgeltlich zugänglich ist» (Art. 10 lit. a aPubV [AS 2004 4934]).

51 Siehe zu den Normen der SNV sogleich IV. 3.2.

52 Botschaft PublG (Fn. 47), S. 7078.

53 Art. 10 lit. a aPubV (Fn. 50). 
sprache erhältlichen Selbstregulierungen nicht mehr weiter stellen dürfte. 54

24 Art. 5 PublG bezieht sich im Übrigen, anders als es der Titel vermuten lässt, nicht per se auf die oben erläuterte Rechtsetzungstechnik der Verweisung, sondern regelt eine besondere Art der Veröffentlichung publikationspflichtiger Texte.55 Das «Verweisungsobjekt» im Sinne von Art. 5 Abs. 1 und 2 PublG ist stets rechtsverbindlich und wird lediglich aus praktischen Gründen nicht in der Form eines Rechtstexts publiziert, sondern eben ausserhalb der AS bzw. der Publikationsplattform. Im Rahmen von Art. 5 PublG ist der durch Verweis veröffentlichte Text dem in der AS veröffentlichen Rechtstext nicht untergeordnet, sondern ebenso rechtsverbindlich wie dieser. ${ }^{6}$ Als allgemeine Rechtsgrundlage für die Veröffentlichung privater Normen ist Art. 5 Abs. 2 PublG folglich erst dann heranzuziehen, wenn die Selbstregulierung, auf die verwiesen wurde, rechtsverbindlich ist.

\section{Beispiele aus der Praxis}

\section{a) SIA-Normen}

Das wahrscheinlich meist genannte Beispiel, wenn von Verweisungen auf private Normen die Rede ist, ist die Normensammlung des SIA. Der SIA ist ein privatrechtlicher Berufs- und Branchenverband, der für seine Mitglieder und sämtliche weiteren Beteiligten ein umfas-

54 Siehe dazu kritisch Uhlmann, Publikation (Fn. 25), S. 90 f.

55 Roth (Fn. 31), S. 180.

56 Lisbeth Sidler, in: Felix Uhlmann/Stefan Höfler (Hrsg.), Gute Gesetzessprache als Herausforderung für die Rechtsetzung, 16. Jahrestagung des Zentrums für Rechtsetzungslehre, Zürich/ St. Gallen 2018, S. 164 f., in Bezug auf Anhänge. sendes Normenwerk geschaffen hat. Sowohl in kantonalen wie auch in bundesrechtlichen Erlassen finden sich Verweise auf die SIA-Ordnungen, so z.B. in einer Fussnote zur Lärmschutzverordnung des Bundes:

Die aufgeführte Norm kann beim Schweizerischen Ingenieur- und Architekten-Verein (SIA), Selnaustrasse 16, 8027 Zürich, kostenlos eingesehen oder unter www.sia.ch gegen Bezahlung bezogen werden. 57

26 Nach dem oben Ausgeführten stellt sich die Frage, ob diese Art der Veröffentlichung in Form eines blossen Verweises in den Fussnoten den dargelegten Publikationserfordernissen zu genügen vermag. Hinzu kommt, dass ein kostenloser Zugang umständlich und ein kostenpflichtiger Zugang - im vorliegenden Fall rund CHF 200.-58 - nicht ganz günstig ist. Dabei ist zunächst festzuhalten, dass die SIA-Normen, ungeachtet eines Verweises in einem staatlichen Erlass, nicht automatisch Geltung erlangen, sondern nur, wenn die SIA-Ordnung wie AGB ausdrücklich Bestandteil des betreffenden privatrechtlichen Vertrags (bspw. eines Architekturvertrags) geworden ist.59 Der von beiden Parteien unterschriebene Vertragstext muss mithin die spezifische SIA-Norm und, aufgrund der periodischen Revisionen, das Erscheinungsjahr

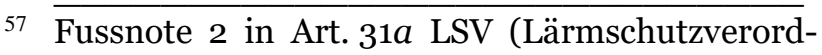
nung vom 15. Dezember 1986 [LSV; SR 814.41]).

58 Vgl. Schallschutz im Hochbau, SIA 181; für SIAMitglieder sind Abonnemente und entsprechende Vergünstigungen vorgesehen.

59 Martin Kurer/Walter Maffioletti/Thomas Spoerri (Hrsg.), Handbuch zum Bauwesen, 2. Aufl., Zürich/St. Gallen 2019, N 2.5; siehe auch Hans Rudolf Spiess/Marie-Theres Huser, Norm SIA 118, Stämpflis Handkommentar, Bern 2014, Einleitung N 21 und 47. 
enthalten, um der SIA-Norm Gültigkeit $\mathrm{zu}$ verleihen. ${ }^{60}$ Insofern kann bei den SIANormen nicht von generell-abstrakten Normen und staatlichem Recht gesprochen werden. Sie entfalten lediglich unter Vertragsparteien, welche die SIA-Norm für anwendbar erklärt haben, eine verbindliche Wirkung. Auch ist der Adressatenkreis der SIA-Normen weitgehend begrenzt, zumal vorwiegend Vertreter der Baubranche davon Gebrauch machen dürften. Bei den SIA-Normen handelt es sich somit um private, technische, branchenspezifische Normenwerke, die äusserst umfangreich und komplex sind und regelmässiger Revisionen bedürfen. Auch mit Blick auf die Ausführungen zu Art. 5 Abs. 2 PublG rechtfertigt sich in einer Gesamtschau die vom Gesetz- bzw. Verordnungsgeber gewählte Form der Publikation inkl. Kostenpflicht beim Online-Bezug durchaus: Selbst bei verbindlichen Normen im Sinne von Art. 5 Abs. 2 PublG genügt die kostenlose Einsichtnahme beim privaten Publikationsorgan den Anforderungen an die Veröffentlichung. Solange ein kostenloser Online-Bezug für verbindliche Normen nicht vorgesehen ist, darf auch an private Normen kein solcher Anspruch gestellt werden.

\section{b) Normen der Schweizerischen Normenvereinigung}

27 In den Fussnoten unterschiedlicher Verordnungen finden sich oftmals auch Verweise auf technische Normen, die bei der SNV bezogen werden können. Die SNV erlässt dabei selbständig auf nationaler Ebene oder in Zusammenarbeit mit

$6 0 \longdiv { \text { BGE } 1 1 8 \text { II } 2 9 5 \text { E. 2a S. 296; BGE } 1 1 7 \text { II } 2 8 2 \text { E. 4b } }$ S. 284; BGE 107 II 172 E. 1c S. 178; Urteile des Bundesgerichts 4C.347/2003 vom 1. April 2004 E. 3.1; 4C.261/2005 vom 9. Dezember 2005 E. 2.3; 4C.371/2006 vom 19. Januar 2007 E. 4; 4A_393/2007 vom 3. Dezember 2007 E. 2.1. europäischen bzw. internationalen Normungsorganisationen technische Normen. ${ }^{61}$

- Die in dieser Verordnung genannten technischen Normen der ISO können beim BAG, 3003 Bern, gratis eingesehen werden. Sie können gegen Entgelt bezogen werden bei der Schweizerischen Normen-Vereinigung, Bürglistrasse 29, 8400 Winterthur; www.snv.ch.62

- $\quad$ Diese technische Norm kann beim Bundesamt für Gesundheit, 3003 Bern, kostenlos eingesehen oder gegen Rechnung bezogen werden bei der Schweizerischen Normen-Vereinigung, Bürglistrasse 29, 8400 Winterthur; www.snv.ch.63

28 Auch bei den Normen, die von der SNV herausgegeben werden, kann zwar festgehalten werden, dass sich deren Beschaffung als aufwendig oder relativ teuer erweisen kann. Gleichzeitig handelt es sich aber um technische Normen, die einen bestimmten und somit begrenzten Anwendungsbereich abdecken $^{64}$ und, wie die SIA-Normen, grundsätzlich nur dann Wirkung entfalten können, wenn die betreffenden Parteien sie explizit als Vertragsbestandteil aufgenommen haben. Insofern scheint auch hier die vom Gesetz- bzw. Verordnungsgeber gewählte Verweisungspraxis

$61 \overline{\text { Siehe «Wer schreibt die Normen?», Beitrag bei }}$ SNV.

62 Fussnote 9 von Art. 10 StSV (Strahlenschutzverordnung vom 26. April 2017 [StSV; SR 814.501]).

63 Fussnote 2 von Art. 6 SLV (Verordnung vom 28. Februar 2007 über den Schutz des Publikums von Veranstaltungen vor gesundheitsgefährdenden Schalleinwirkungen und Laserstrahlen [Schall- und Laserverordnung, SLV; SR 814.49]).

64 Wie z.B. die SN EN 71-14 «Sicherheit von Spielzeug - Teil 14: Trampoline für den häuslichen Gebrauch». 
aus rechtsstaatlicher Hinsicht weitgehend problemlos zu sein. Zudem scheint der Gesetzgeber, zumindest wenn man der Botschaft zum aktuellen Publikationsgesetz folgen mag, davon auszugehen, dass gerade die Normen der SNV unter Art. 5 Abs. 2 PublG fallen. ${ }^{6}$ Entsprechend kann auf weitere Legitimitätsüberlegungen verzichtet werden, zumal die Normen der SNV aufgrund der Möglichkeit der kostenlosen Einsichtnahme bei der zuständigen Bundesbehörde den gesetzlichen Anforderungen von Art. 5 Abs. 2 PublG zu genügen vermögen.

\section{c) Publikationen der Schweizeri- schen Akademie der Medizini- schen Wissenschaften (SAMW)}

29 Nicht alle privaten Normenerzeuger tun sich schwer mit dem Zugang zu ihren Normen. ${ }^{66}$ Die medizin-ethischen Richtlinien der SAMW sind in allen drei Landessprachen sowie in Englisch verfügbar, können auf der Website des Vereins kostenlos heruntergeladen und in gedruckter Form («auch in grösseren Mengen») bestellt werden. ${ }^{67}$ In der Praxis kommt den Richtlinien durch ihre Aufnahme in die Standesordnung der $\mathrm{FMH}^{68}$ eine grosse Bedeutung zu, zumal sie auf diese Weise gegenüber allen in der Schweiz tätigen Ärztinnen und Arzte verbindliche Wirkung entfalten.

\section{d) Kostenpflichtige Normen des Bundes}

3o Anhand dreier Beispiele soll nachfolgend gezeigt werden, dass nicht nur private

\footnotetext{
65 Siehe oben IV. 2.; Botschaft PublG (Fn. 47), S. 7078.

66 Vgl. auch die durchwegs kostenlos einsehbaren Richtlinien im Bereich des Finanzmarktrechts.

67 Siehe Publikation der SAMW über medizinischethische Richtlinien.

68 Verbindung der Schweizer Ärztinnen und Ärzte.
}

Normenerzeuger versucht sind, für die von ihnen verfassten Regelwerke eine Gebühr zu verlangen.

- In der Hochschulbauten-BeitragsVerordnung 69 wird in Art. 2 auf die Normen und Empfehlungen des Bundesamtes für Sport verwiesen und in der Fussnote festgehalten, dass diese beim Bundesamt für Sport unter www.basposhop.ch kostenpflichtig bezogen werden können. Alternativ können die Normen und Empfehlungen auch kostenlos beim Staatssekretariat für Bildung, Forschung und Innovation, Einsteinstrasse 2, 3003 Bern eingesehen werden. Dabei ergibt sich beim kostenpflichtigen Bezug auf der Website des BaSpo eine Gebühr von CHF 12.- bis 60.- pro gedruckte Broschüre. Immerhin können die Broschüren auch kostenlos heruntergeladen und vervielfältigt werden.

- Auch die Pharmacopoea Helvetica, welche Qualitätsvorschriften für Arzneimittel und pharmazeutische Hilfsstoffe sowie für einzelne Medizinprodukte enthält und von Swissmedic ${ }^{70}$ vertrieben wird, findet sich in Fussnoten unterschiedlicher Erlasse wieder:

Die Pharmacopoea Helvetica wird von der Swissmedic herausgegeben und kann beim BBL, Verkauf Bundespublikationen, 3003 Bern, www.bundespublikationen.admin.ch,

$69 \overline{\text { Verordnung des WBF (Eidgenössisches Depar- }}$ tement für Wirtschaft, Bildung und Forschung) vom 23. November 2016 über die Bauinvestitions- und Baunutzungsbeiträge für Hochschulbauten (HSBBV; SR 414.201.1).

70 Schweizerisches Heilmittelinstitut, eine öffentlich-rechtliche Anstalt des Bundes. 
zu den in der GebV-Publ vorgesehenen Bedingungen bezogen werden (SR 172.041.11).71

Aktuell wird für den Bezug der
Pharmacopoea Helvetica ein Betrag
von CHF 105. - verlangt.72

- Ähnlich kostenpflichtig waren auch die, im Vergleich zu den eben genannten Beispielen in der Praxis äusserst relevanten, SKOS73-Richtlinien.74 Seit Anfang 2013 können die Richtlinien auf der Website der SKOS zumindest kostenlos heruntergeladen werden; die Kosten für die Papierversion belaufen sich auf CHF 55.-.

31 Klarerweise kann - mit Ausnahme der SKOS-Richtlinien - auch im Falle des Bundes und der bundesnahen Institutionen wie oben argumentiert werden, dass die betreffenden Normen einen eingeschränkten Adressatenkreis haben, kaum neue Rechte und Pflichten für die Adressaten enthalten und, insbesondere im Falle der Pharmacopoea Helvetica, einen derart umfangreichen, detaillierten und fachspezifischen Inhalt aufweisen, dass sich die Kostenpflichtigkeit bis zu einem gewissen Ausmass, rechtfertigt. Zudem fällt die Pharmacopoea Helvetica als Erlass der Swissmedic unter Art. 2 lit. e PublG sowie in den Anwendungsbereich von Art. 5 Abs. 1 PublG. Immerhin sollte auch

71 Fussnote $\mathrm{zu}$ Anhang 2 der ArzneimittelBewilligungsverordnung (Verordnung vom 14. November 2018 über die Bewilligungen im Arzneimittelbereich [SR 812.212.1]).

72 Auf der Website der Bundespublikationen.

73 Schweizerische Konferenz für Sozialhilfe; Richtlinien der SKOS.

74 Siehe auch Felix Uhlmann/Iris Binder, Verwaltungsverordnungen in der Rechtsetzung; Gedanken über Pechmarie, LeGes 2009/2 S. 151 f., $165 \mathrm{f}$. der Bund als Normerzeuger, insbesondere wenn er für die Herausgabe von Normen und Richtlinien eine Gebühr erhebt und damit «unternehmerisch» tätig wird, jeweils im Einzelfall abwägen, ob und in welcher Höhe sich eine Gebühr rechtfertigt.

\section{Exkurs: Urheberrecht}

32 Anders als private Normenwerke sind Gesetze, Verordnungen, völkerrechtliche Verträge und andere amtliche Erlasse nach Art. 5 Abs. 1 lit. a URG75 urheberrechtlich nicht geschützt. Fraglich ist nun, ob staatlich gesteuerte Selbstregulierungen noch urheberrechtlichen Schutz geniessen oder aufgrund ihrer Nähe zum staatlichen Recht diesem auch aus urheberrechtlicher Sicht gleichgestellt wird. In der Lehre wird davon ausgegangen, dass private Normen so lange urheberrechtlich geschützt sind, als das staatliche Recht nicht auf sie verweist. Sobald Gesetz- oder Verordnungsgeber einen Verweis auf private Normen anbringen und den privaten Normen auf diese Weise eine rechtliche Verbindlichkeit zukommt, fallen sie unter den Anwendungsbereich von Art. 5 URG. ${ }^{76}$

\section{Fazit}

33 Es sollte zumindest davon ausgegangen werden können, dass, soweit in der AS auf eine Norm bzw. eine Normensammlung verwiesen wird, dieser eine gewisse Verbindlichkeit zukommen soll. Die mittels Verweis in die Nähe des staatlichen Rechts geführte Norm wird nicht nur pu-

$7 5 \longdiv { \text { Bundesgesetz vom 9. Oktober } 1 9 9 2 \text { über das Urhe- } }$ berrecht und verwandte Schutzrechte (Urheberrechtsgesetz, URG; SR 231.1).

76 Reto M. Hilty, Urheberrecht, Bern 2011, N 129; Manfred Rehbinder/Adriano Viganò, URG-Kommentar, Zürich 2008, Art. 5 N 2. 
bliziert, sondern erfüllt durch die Nennung in Gesetz oder Verordnung auch die Voraussetzungen ihrer Anwendbarkeit und Verbindlichkeit. Oder anders gesagt: Verweisung und deren Publikation sind die Instrumente des Staates, um einer ursprünglich privaten Norm Verbindlichkeit und Allgemeingültigkeit zu verleihen. Entsprechend sollte sie auch publiziert werden. Das geltende Publikationsrecht bietet denn auch die erforderlichen Rahmenbedingungen, so dass die Praxis des Bundes, vermehrt auf private Normen zu verweisen, durchaus gerechtfertigt ist. Soweit zumindest die Möglichkeit des kostenlosen Zugangs zu den privaten Normen in Form der Einsichtnahme vor Ort gewährleistet ist, ist nach der hier vertretenen Ansicht auch gegen eine Kostenpflicht beim Online-Bezug nichts einzuwenden. Nichtsdestotrotz wäre eine einheitliche Gesetzgebung zur Selbstregulierung im Allgemeinen wünschenswert und auch im Hinblick auf deren Publikation von Vorteil. 